\title{
EFEITOS DA ADUBAÇÃo NITROGENADA E POTÁSSICA NA PRODUÇÃo E NA QUALIDADE DE FRUTOS DE LARANJEIRA-'VALÊNCIA'
}

\author{
MARCELO CARMINATI DE ALMEIDA², JOSÉ GERALDO BAUMGARTNER ${ }^{3}$
}

\begin{abstract}
RESUMO - Os critérios para recomendação de adubação nitrogenada e potássica em pomares cítricos carecem de experimentação regional. O objetivo deste experimento foi avaliar os efeitos de doses combinadas de $\mathrm{Ne}$ de $\mathrm{K}_{2} \mathrm{O}$ para a laranjeira-Valência cultivada em solo representativo do município de Adolfo, Norte do Estado de São Paulo, de citricultura desenvolvida. Em delineamento tipo fatorial 3 × 3, com 3 repetições, foram combinadas as doses de 94; 188 e $376 \mathrm{~kg} \mathrm{ha}^{-1}$ de N, como nitrato de amônio e $38 ; 75$ e $150 \mathrm{~kg} \mathrm{ha}^{-1}$ de $\mathrm{K}_{2} \mathrm{O}$, como cloreto de potássio, durante três safras (1997 a 1999). Anualmente, foram feitas avaliações de diagnose foliar para nitrogênio e potássio, de produção e de análise de suco para acidez titulável e teor total de sólidos solúveis. Os dados obtidos mostraram que os pomares mantiveram a produtividade com as doses mínimas de $\mathrm{N}$ e de $\mathrm{K}_{2} \mathrm{O}$ durante os três anos, não havendo resposta em produção às doses crescentes desses nutrientes. Os teores foliares de $\mathrm{N}$ e de $\mathrm{K}$ mantiveram-se dentro das faixas consideradas adequadas, nos tratamentos com doses mínimas de $\mathrm{N}$ e de $\mathrm{K}_{2} \mathrm{O}$, nas três safras. Apenas no segundo ano, em que a produção foi diminuída por fatores climáticos, foram observados efeitos significativos de combinações de doses de $\mathrm{N}$ e de $\mathrm{K}_{2} \mathrm{O}$ sobre a acidez e o teor de sólidos solúveis do suco.
\end{abstract}

Termos para indexação: citros, nitrogênio, potássio, adubação.

\section{EFFECTS OF NITROGEN AND POTASSIUM FERTILIZATION ON PRODUCTION AND FRUIT QUALITY OF CITRUS ' VALENCIA'}

\begin{abstract}
Nitrogen and potassium fertilization criteria for citrus orchards was not clearly stablished yet, based on regional experimentation. This experiment was carried out in order to compare the effects of combined levels of $\mathrm{N}$ and $\mathrm{K}$ fertilization on citrus orchard of 'Valência', cultivaded in Adolfo (Northen of São Paulo State - Brazil). Three levels of nitrogen, 94, 188 and $376 \mathrm{~kg} \cdot \mathrm{ha}^{-1}$ as ammonium nitrate and three levels of potassium, 38,75 and $150 \mathrm{~kg} \mathrm{ha}^{-1}$ of $\mathrm{K}_{2} \mathrm{O}$ as potassium chloride, were combined in factorial design $3 \times 3$ with 3 replications, during three harvest (1997 to 1999). Fruit productions, fruit tecnological analysis and foliar diagnosis were evaluated yearly. Data showed that the trees kept the productivity with the mininum levels of $\mathrm{N}$ and $\mathrm{K}_{2} \mathrm{O}$ for three years and no response in productivity was observed in this period. Only in the second year, when yield was decreased by climatic factors, it was observed effects of combined levels of $\mathrm{N}$ and $\mathrm{K}_{2} \mathrm{O}$ on juice acidity and soluble solids contents.
\end{abstract}

Index terms: citrus, nitrogen, potassium, fertilization.

O nitrogênio é o problema central na recomendação de adubos na citricultura, pois é complexa a avaliação da ciclagem desse elemento no ambiente do pomar. A análise de fertilidade do solo não permite estimativa de disponibilidade de nitrogênio, e a análise do teor total deste nas folhas também tem sido questionada como critério diagnóstico. As plantas, de um modo geral, exigem potássio na medida da capacidade de metabolização do nitrogênio. Na citricultura, a relação N/K nos tecidos foliares afeta a produção e a qualidade dos frutos como já observaram diferentes pesquisadores (Reese \& Koo, 1975; DuPlessis \& Koen, 1988).

O objetivo deste experimento foi avaliar efeitos de doses combinadas de nitrogênio e potássio sobre a produção e a qualidade dos frutos de laranjeira-Valência, cultivada no município de Adolfo, Norte do Estado de São Paulo. O solo, Podzólico
Vermelho-Amarelo eutrófico, textura arenosa a média, em relevo suavemente ondulado, foi coletado em amostras compostas, nas faixas adubadas e nas profundidades de $0-20 \mathrm{~cm}$ e $20-40 \mathrm{~cm}$ que foram submetidas à análise de fertilidade, segundo os métodos descritos por Raij \& Quaggio, 1983. Os resultados analíticos estão na Tabela 1. Com base na análise de solo, estimativa de safra e análises foliares anteriores, foram estabelecidas as doses de $\mathrm{N}$ $\left(\mathrm{kg} \mathrm{ha}^{-1}\right): \mathrm{N}_{1}=94 ; \mathrm{N}_{2}=188$ e $\mathrm{N}_{3}=376$ e de $\mathrm{K}_{2} \mathrm{O}\left(\mathrm{kg} \mathrm{ha}^{-1}\right): \mathrm{K}_{1}=38$; $\mathrm{K}_{2}=75$ e K $3=150$. As parcelas foram compostas por 3 linhas de 6 plantas, considerando-se como área útil as quatro plantas centrais. O pomar foi plantado no espaçamento de $8 \times 4 \mathrm{~m}$ de forma que cada parcela tinha área de $576 \mathrm{~m}^{2}$. O delineamento experimental adotado foi tipo fatorial $3 \times 3$, em blocos ao acaso, com 3 repetições. Os adubos utilizados foram o nitrato de amônio $(34 \%$ de $\mathrm{N})$ e o cloreto de potássio $\left(58 \%\right.$ de $\left.\mathrm{K}_{2} \mathrm{O}\right)$, granulados. A

1 (Trabalho 061/2001). Recebido: 12/03/2001. Aceito para publicação: 08/02/2002.

2 Eng $^{\circ}$ Agr $^{\circ}$ Mestre em Produção Vegetal (FCAV-UNESP. Câmpus de Jaboticabal). Rua Prudente de Moraes 45, Apt ${ }^{\circ}$ 51, Caixa Postal 29. $14730-$ 000. Monte Azul Paulista, SP.

3 Professor Titular do Departamento de Solos e Adubos da FCAV-UNESP, Câmpus de Jaboticabal. Via de acesso Prof. Paulo Donato Castellane S/ N. 14884-900. Jaboticabal, SP. 
TABELA 1 - Análise prévia de fertilidade de amostras coletadas nas faixas adubadas.

\begin{tabular}{|c|c|c|c|c|c|c|c|}
\hline \multirow{2}{*}{$\begin{array}{c}\text { Profundidades } \\
\text { c m }\end{array}$} & \multirow{2}{*}{$\begin{array}{c}\mathrm{pH} \\
\mathrm{C} \mathrm{aC} 1_{2}\end{array}$} & \multirow{2}{*}{$\begin{array}{c}\mathrm{P} \\
\mathrm{mg} \mathrm{d} \mathrm{m}\end{array}$} & $\mathrm{K}$ & $\mathrm{Ca}^{2+}$ & $\mathrm{Mg}^{2-}$ & $\mathrm{H}+\mathrm{A} 1$ & \multirow{2}{*}{ V\% } \\
\hline & & & & $\mathrm{m} \mathrm{m}$ & & & \\
\hline $0-20$ & 5,6 & 20 & 4,0 & 29 & 8 & 17 & 70 \\
\hline $20-40$ & 5,1 & 5 & 1,7 & 24 & 13 & 18 & 68 \\
\hline
\end{tabular}

TABELA 2 - Produção de frutos (kg/planta) de laranjeira-Valência.

\begin{tabular}{ccccc}
\hline Doses $\left(\mathrm{kg} \mathrm{ha}^{-1}\right)$ & \multicolumn{3}{c}{ Produção $(\mathrm{kg} / \mathrm{planta})$} & \multirow{2}{*}{ M édias } \\
\cline { 1 - 4 } Nitrogênio $(\mathrm{N})$ & 1997 & 1998 & 1999 & 172 \\
94 & 205 & 94 & 216 & 179 \\
188 & 208 & 104 & 225 & 172 \\
376 & 209 & 97 & 211 & 175 \\
Potássio $\left(\mathrm{K}_{2} \mathrm{O}\right)$ & & & & 177 \\
38 & 199 & 103 & 223 & 171 \\
75 & 213 & 97 & 220 & - \\
150 & 209 & 95 & 210 & - \\
F (doses de N) & 0,04 & 0,60 & 0,72 & - \\
F doses de $(\mathrm{K})$ & 0,39 & 0,38 & 0,60 & - \\
F (N K $)$ & 0,06 & 1,92 & 0,46 & 12,21 \\
CV $(\%)$ & 15,81 & 19,88 & & \\
\hline
\end{tabular}

TABELA 3 - Teores totais de N e K nas terceiras e quartas folhas de ramos frutíferos.

\begin{tabular}{|c|c|c|c|c|c|c|}
\hline \multicolumn{7}{|c|}{ Teores totais na matéria seca $\left(\mathrm{g} \mathrm{kg}^{-1}\right)$} \\
\hline Doses $\left(\mathrm{kg} \mathrm{ha}^{-1}\right)$ & \multicolumn{2}{|c|}{1997} & \multicolumn{2}{|c|}{1998} & \multicolumn{2}{|c|}{1999} \\
\hline Nitrogênio $(\mathrm{N})$ & $\mathrm{N}$ & $\mathrm{K}$ & $\mathrm{N}$ & $\mathrm{K}$ & $\mathrm{N}$ & $\mathrm{K}$ \\
\hline 94 & 27,0 & 13,4 & $24,8 \mathrm{~b}$ & 10,2 & $25,1 \mathrm{~b}$ & 12,5 \\
\hline 188 & 27,2 & 12,8 & $25,1 \mathrm{ab}$ & 9,1 & $28,5 \mathrm{a}$ & 11,6 \\
\hline 376 & 27,3 & 13,7 & $26,6 \mathrm{a}$ & 10,2 & 28,3 a & 10,5 \\
\hline \multicolumn{7}{|l|}{ Potássio $\left(\mathrm{K}_{2} \mathrm{O}\right)$} \\
\hline 38 & 26,8 & 13,4 a & 25,6 & 10,1 & 27,7 & 10,7 \\
\hline 75 & 27,3 & 14,2 a & 25,8 & 10,0 & 27,7 & 11,6 \\
\hline 150 & 27,3 & $12,4 \mathrm{~b}$ & 25,2 & 9,5 & 26,5 & 12,3 \\
\hline $\mathrm{F}(\operatorname{doses} \mathrm{de} N)$ & 0,13 & 3,29 & $4,69 *$ & 3,19 & $5,93 *$ & 0,78 \\
\hline$F(\operatorname{doses} d e K)$ & 0,73 & $12,38 * *$ & 0,43 & 0,91 & 0,84 & 0,54 \\
\hline $\mathrm{F}(\mathrm{N} \times \mathrm{K})$ & 1,22 & 1,92 & 1,11 & 0,96 & 0,37 & 0,88 \\
\hline
\end{tabular}

Letras diferentes nas colunas significam médias diferentes, pelo teste de Tukey, a $5 \%$.

TABELA 4 - Acidez e teor de sólidos solúveis do suco de frutos de laranjeira-'Valência'.

\begin{tabular}{|c|c|c|c|}
\hline $\begin{array}{l}\text { Doses de } \mathrm{N} \\
\left(\mathrm{kg} \mathrm{ha}^{-1}\right)\end{array}$ & $\begin{array}{c}\text { Doses de } \mathrm{K}_{2} \mathrm{O} \\
\mathrm{kg} \mathrm{ha}^{-1}\end{array}$ & $\frac{\text { Acidez }(\% \text { ac.cítrico })}{\text { Agosto } / 98}$ & $\frac{\text { Sol. solúveis }\left({ }^{0} \mathrm{Brix}\right)}{\text { Setembro } / 98}$ \\
\hline 94 & 38 & $1,03 \mathrm{~b}$ & 10,39 a \\
\hline 94 & 75 & $0,99 \mathrm{~b}$ & $10,06 \mathrm{~b}$ \\
\hline 94 & 150 & $1,15 \mathrm{a}$ & 10,45 a \\
\hline 188 & 38 & $1,13 \mathrm{a}$ & $10,51 \mathrm{a}$ \\
\hline 188 & 75 & $1,07 \mathrm{ab}$ & $10,31 \mathrm{ab}$ \\
\hline 188 & 150 & $1,06 \mathrm{~b}$ & $10,71 \mathrm{a}$ \\
\hline 376 & 38 & $1,06 \mathrm{~b}$ & $10,40 \mathrm{a}$ \\
\hline 376 & 75 & $1,17 \mathrm{a}$ & $10,83 \mathrm{a}$ \\
\hline 376 & 150 & $1,12 \mathrm{ab}$ & $10,27 \mathrm{ab}$ \\
\hline $\mathrm{F}(\mathrm{N} \times \mathrm{K})$ & & $3,05 *$ & $3,40 *$ \\
\hline dms (Tukey $5 \%)$ & & 0,08 & 0,32 \\
\hline $\mathrm{CV}(\%)$ & & 6,45 & 2,59 \\
\hline
\end{tabular}


adubação foi feita em faixas laterais, dos dois lados das plantas, em três parcelas, na época de chuvas nos anos de 1996 a 1999. Nos meses de março de cada ano, cerca de 40 dias após a última parcela de adubação, foram coletadas amostras de folhas para fins de diagnose foliar. As amostras foram preparadas e analisadas para teores totais de nitrogênio e de potássio conforme métodos descritos por Bataglia et al., 1983. Nos meses de agosto, setembro, outubro e novembro de cada ano, foram coletadas amostras de 48 frutos por parcela, determinando-se, nos mesmos, a acidez titulável e o teor de sólidos solúveis ( ${ }^{\circ}$ Brix) segundo métodos descritos por Reed et al., 1986. Em novembro de cada ano, foi feita a colheita total de frutos maduros, determinando-se o peso dos mesmos por parcela (Tabela 2). Esses dados mostram que não houve efeitos significativos das doses combinadas de nitrogênio e potássio na produção da laranjeira-Valência durante três safras consecutivas. As produções nos anos de 1997 e 1999 foram normais, de acordo com os padrões da laranjeira-"Valência" indicados por Figueiredo (1991). No ano de 1998, a produção caiu pela metade devido à irregularidade de chuvas, afetando o florescimento e o pegamento dos frutos. Os dados do primeiro ano de não-resposta à adubação NK são considerados previsíveis em função do histórico do pomar. Já no segundo ano, a produção foi prejudicada por condições climáticas desfavoráveis e podese inferir que a colheita obtida pouco exigiu quanto à disponibilidade de nutrientes. A não-resposta à adubação NK foi inesperada na terceira safra, pois pode-se considerar as doses menores $\left(\mathrm{N}_{1}\right.$ e $\left.\mathrm{K}_{1}\right)$, como uma subadubação, visto que as doses centrais $\mathrm{N}_{2}$ e $\mathrm{K}_{2}$ foram baseadas nas recomendações do Grupo Paulista (1994) e, portanto, as doses $\mathrm{N}_{3}$ e $\mathrm{K}_{3}$ podem ser consideradas excessivas. Entretanto, as plantas mantiveram a mesma produtividade, quer recebendo 94 e $38 \mathrm{~kg} \mathrm{ha}^{-1}$ de $\mathrm{N}$ e de $\mathrm{K}_{2} \mathrm{O}$, quer 376 e $150 \mathrm{~kg} \mathrm{ha}^{-1}$ de $\mathrm{N}$ e de $\mathrm{K}_{2} \mathrm{O}$, respectivamente, por três anos consecutivos. Este contraste confirma a capacidade de as plantas cítricas bem conduzidas manterem a produtividade às custas de suas próprias reservas e das do solo por período de tempo relativamente longo. Os dados da Tabela 3 mostram que os teores foliares de nitrogênio e potássio foram pouco afetados pelos tratamentos de adubação, ocorrendo pequenas oscilações dentro das faixas consideradas adequadas $\left(\mathrm{N}=23-27 \mathrm{~g} \mathrm{~kg}^{-1} \mathrm{e} \mathrm{K}\right.$ $=10-15 \mathrm{~g} \mathrm{~kg}^{-1}$ ).Pode-se verificar, ainda, que $\mathrm{o}$ ano agrícola teve maior influência nos teores foliares de $\mathrm{N}$ e de $\mathrm{K}$ que os níveis de adubação. Todavia, os teores foliares de $\mathrm{N}$ aumentaram significativamente em função das doses crescentes do nutriente, apenas nos anos de 1998 e 1999. A variação obtida sobre os teores foliares de $\mathrm{K}$, em função de doses do nutriente, foi estranha, pois constatou-se diminuição no teor foliar com aumento da dose, apenas no primeiro ano. Isso, entretanto, é passível de ocorrer, por dois fatores não excludentes: diluição do nutriente em copa de maior vegetação e concentração salina na faixa adubada, diminuindo a absorção via radicelas.

Das análises tecnológicas dos frutos, constataram-se efeitos esparsos dos tratamentos sobre a acidez (gramas de ácido cítrico / $100 \mathrm{~mL}$ de suco) e teor de sólidos solúveis ( ${ }^{\circ}$ Brix). Os efeitos significativos, identificados pelo teste $\mathrm{F}$, foram desdobrados e as médias comparadas pelo teste de Tukey a 5\% (Tabela 4). Os dados mostram que apenas na safra de menor produção, em razão de fatores climáticos (1998), ocorreram efeitos de doses de $\mathrm{N}$ e $\mathrm{K}$ sobre as características de acidez e teor total de sólidos solúveis. Houve interação significativa entre doses de N e K para ambas as variáveis. Dessa forma, a maior dose de $\mathrm{K}\left(150 \mathrm{~kg} \mathrm{ha}^{-1} \mathrm{de}_{2} \mathrm{O}\right)$, comparada às outras, causou aumento da acidez do suco apenas dentro da menor dose de nitrogênio (94 $\left.\mathrm{kgha}^{-1} \mathrm{de} \mathrm{N}\right)$, ao passo que a dose intermediária de $\mathrm{K}\left(75 \mathrm{~kg} \mathrm{ha}^{-1}\right.$ de $\mathrm{K}_{2} \mathrm{O}$ ) causou diminuição no teor de sólidos solúveis, apenas dentro da maior dose de nitrogênio. Esses efeitos foram presentes só nas primeiras amostragens de frutos, isto é, sobre a acidez nas amostras de frutos coletados na mês de agosto e sobre o teor total de sólidos solúveis, na amostragem de setembro. O efeito de doses de K sobre a acidificação do suco está coerente com o que já foi observado por diferentes pesquisadores (Reese \& Koo, 1975). Pode-se observar ainda que, fixando a dose intermediária de potássio $\left(\mathrm{K}_{2}=75 \mathrm{~kg} \mathrm{ha}^{-1} \mathrm{de} \mathrm{K}_{2} \mathrm{O}\right)$, à medida que aumentou a dose de $\mathrm{N}$, aumentou também o teor de sólidos solúveis do suco. Diferentes autores têm considerado como inconsistentes os efeitos de doses de nitrogênio sobre o teor de sólidos do suco (Reese \& Koo, 1975; Embleton et al., 1973).

\section{REFERÊNCIAS BIBLIOGRÁFICAS}

BATAGLIA, O. C; FURLANI, A. M. C; TEIXEIRA, J. P. F; FURLANI, P. R.; GALLO, J. R. Métodos de análises químicas de plantas. Campinas: Instituto Agronômico, 1983. 48p. (Boletim Técnico, 78).

DuPLESSIS, S. F; KOEN, T. J. The effect of N and K fertilization on yield and fruit size of 'Valência': In: INTERNATIONAL CITRUS CONGRESS, 6., 1988, Israel. Proceedings. P. 663-672.

EMBLETON, T. W; WINSTON, W. J; LABANAUSKAS, C. K; REUTHER, W. Leaf analysis as a diagnostic tool and guide to fertilization. In: REUTHER, W, BATCHELOR, L. D, WEBBER, H. $\mathrm{J}(\mathrm{Ed})$. The citrus industry. Berkeley: University of Califórnia, Division of Agricultural Sciences, 1973, v. 3, p. 183-210.

FIGUEIREDO, J. O. Variedades-copa de valor comercial. In: RODRIGUEZ, O; VIEGAS, F; POMPEU JR, J; AMARO, A. S. (ed). Citricultura Brasileira 2. ed. rev. ampl. Campinas: Fundação Cargill 1991.v 1, 492p.

GRUPO PAULISTA DE ADUBAÇÃO E CALAGEM PARA CITROS. Recomendações de adubação e calagem para citros no Estado de São Paulo. 3. ed. rev. atual. Cordeirópolis: Laranja, 1994. 27p. Edição especial.

RAIJ, B. van; QUAGGIO, J. A. Métodos de análises de solo para fins de fertilidade. Campinas: Instituto Agronômico, 1983.31 p. (Boletim Técnico, 81).

REED, J. B; HENDRIX, J. R, C. M; HENDRIX, D. L. Quality control manual for citrus processing plantas. Flórida: Intercit, 1986.250p. REESE, R. L; KOO, R. G. J. Effects of N and K fertilization on internal fruit quality of three major Flórida oranges cultivars. J. Am. Soc. Hort. Sci. no 100, v 2, p.425-428, 1975. 